\title{
UNA PRESENCIA NO TAN SINGULAR: ESPAÑOLES EN LA ECONOMIA DEL OPIO EN ASIA ORIENTAL (1815-1843)
}

\section{NOT SUCH AN EXCEPTIONAL PRESENCE: SPANIARDS IN THE OPIUM BUSINESS IN EAST ASIA (1815-1843)}

\author{
Ander Permanyer Ugartemendia \\ Institut Universitari d'Història Jaume Vicens i Vives, \\ Universitat Pompeu Fabra, Barcelona
}

\section{RESUMEN}

En este texto analizamos la presencia española en el comercio cantonés y, por extensión, de Asia oriental, una presencia no tan singular, heredera de actividades previas y en interacción con individuos, redes y corporaciones de otras nacionalidades. Identifica sus vínculos con el imperio español -con la plata, y con el crédito y productos filipinos-, además del protagonismo de individuos - predominantemente vascos-, con sus redes en el Índico y el Pacífico, así como destaca el papel de la desconocida Compañía de Filipinas. Asimismo, señala el protagonismo de la economía filipina en Asia, enfatizando su conexión con el negocio del opio y el comercio cantonés, fruto de las iniciativas criollas filipinas tras el fin del Galeón de Manila. Detallando intereses conjuntos de españoles y británicos -principalmente escoceses-, además, la tesis apunta hacia las recientes redefiniciones del "Canton system", que ponen de relieve el predominio de la colaboración, más que de la confrontación, en su desarrollo.

Palabras clave: opio; Real Compañía de Filipinas, Jardine, Matheson \& Co., Yrisarri y $C^{a}$; Lorenzo Calvo y Mateo

\begin{abstract}
This text analyses the Spanish presence in Cantonese trade and, by extension, in East Asia. It was not such an exceptional presence and was the result of previous activities and interaction with individuals, networks and corporations of other nationalities. It identifies links with the Spanish empire (with silver, Philippine credit and products), in addition to the leading role of certain individuals (mainly Basques) with their networks in the Indian and Pacific Oceans. It also highlights the role of the unknown Royal Philippine Company. Likewise, it spotlights the leading role of the Philippine economy in Asia, emphasising its connection with the opium business and Cantonese trade, as a result of Philippine Creole initiatives following the end of the Manila Galleon. Detailing common Spanish and British (mainly Scottish) interests, the article also makes reference to recent redefinitions of the "Canton System", which highlight the predominance of collaboration, rather than confrontation, during its development.
\end{abstract}

Keywords: opium, Royal Philippine Company, Jardine, Matheson \& Co.; Yrisarri \& Co.; Lorenzo Calvo y Mateo 


\section{Presentación}

La participación española en el negocio del opio en China, a principios del siglo XIX, ha sido objeto de un importante vacío historiográfico, tanto en los análisis del comercio cantonés como en los de la presencia española en Asia y Pacífico. En efecto, hubo una entrada de intereses españoles - predominantemente vascos- en dicho sector, que se desarrolló entre Bengala y el sur de China, que si bien fue breve en el tiempo -entre 1815 y $1830-$, tuvo una importante relevancia no tan sólo por lo que representó en el momento, sino también por sus repercusiones posteriores. La tesis doctoral "La participación española en la economía del opio en Asia Oriental tras el fin del Galeón" (PERMANYER UGARTEMENDIA, 2013) identifica, describe y analiza el significado de dichas actividades.'

La última generación de los empleados de la Real Compañía de Filipinas (en adelante, RCF ) en Calcuta (Kolkata) y Cantón (Guangzhou), que ocuparon sus puestos desde la segunda mitad de la década de 1810 hasta el desmantelamiento de la Compañía, se dedicará de lleno al comercio del opio, tras las incursiones puntuales de sus predecesores al frente de dichas factorías durante las dos primeras décadas del siglo XIX. El sector estaba entonces en pleno proceso de transición y crecimiento exponencial, con un protagonismo indiscutible de las firmas privadas británicas, junto las cuales también prosperaron las iniciativas privadas formadas por los empleados de la RCF, colaborando estrechamente con aquéllas, y con el apoyo financiero procedente de Filipinas. Españoles y británicos desarrollarán conjuntamente estrategias y prácticas, que serán continuadas en décadas subsiguientes. 
En este artículo presentaremos la tesis doctoral mencionada, centrándonos en la participación más activa de la década de 1820, así como indicaremos los elementos propios de las redes españolas del opio que, además, persistieron hasta la primera guerra del opio (1839-42). Analizaremos en primer lugar las condiciones de dicho comercio, para pasar a detallar las redes españolas que se dedicaron a él, indicando las fuentes utilizadas y los estudios hechos hasta la fecha. Finalmente, destacaremos tres elementos definitorios de estas redes españolas: su papel en un contexto de continuo cambio en Asia oriental, su estrecha colaboración con los británicos, y el protagonismo indiscutible de Filipinas al respecto.

\section{La "Cuestión del OPio"}

El opio es uno de los protagonistas principales de las economías políticas de Asia oriental en el siglo XIX, símbolo del "siglo de humillación" chino y objeto de grandes equívocos y revisiones recientes. La pasta resultante de la savia de la cebolla de la adormidera era ya objeto de comercio en el Índico y los mares de Asia oriental a la llegada de los europeos a principios del siglo $\mathrm{XVI}$, quienes paulatinamente irán haciéndose con distintas áreas de su producción y distribución. Finalmente, los británicos acabarán por dominarlo, tanto en su producción en la India, con la consolidación de su cultivo y de su elaboración en las factorías de Patna (Bihar) y Ghazipur (Uttar Pradesh) durante el último tercio del siglo XVIII, como en gran parte de su distribución, que se delegaba a terceros en las subastas de Calcuta y Bombay (BOUCHON, 1991, PRAKASH, 1987, TROCKI, 1999). El opio será algo más que un negocio lucrativo, pieza clave para el apoyo de los imperialismos europeos en Asia (TROCKI, 1999, DERKS, 2012); de este modo, representará, por ejemplo, un $20 \%$ de las finanzas de la India británica en el conjunto de su existencia. ${ }^{2}$ El opio era una mercancía estratégica para el sostenimiento del comercio sino-indio, vital para las finanzas de la Compañía Británica de las Indias Orientales (East India Company, en adelante, $E(C)$, cada vez más presionada por las mayores obligaciones resultantes del gobierno de la India. Desde el último tercio del siglo XVIII, tanto el algodón en rama como el opio compensarán el balance deficitario de dicho comer-

2 "I argue [...] that without the drug, there probably would have been no British Empire" (TROC$\mathrm{KI}, 1999$, p. XIII). 
cio, que se había agravado a raíz del crecimiento de las inversiones en té tras la Commutation Act de 1784, que reducía drásticamente sus tasas de entrada en Inglaterra (MUI - MUI, 1984).

El crecimiento de las importaciones de opio en el sur de China dan una idea del aumento del consumo. Éste creció claramente durante el último tercio del siglo XVIII, para mantenerse estable durante las dos primeras décadas del siglo XIX -con una media de unos 4.200 cajones por temporada entre 1800 y $1821-$, y dispararse desde 1820, como puede verse en la tabla siguiente:

\section{Importaciones de opio en Cantón (en cajones) ${ }^{3}$}

\begin{tabular}{|c|r|}
\hline 1770 & 700 \\
\hline 1780 & $1.200-1.500$ \\
\hline 1790 & $\sim 4.000$ \\
\hline 1800 & 4.570 \\
\hline $1820-21$ & 4.244 \\
\hline $1821-22$ & 5.459 \\
\hline $1822-23$ & 7.773 \\
\hline
\end{tabular}

\begin{tabular}{|r|r|}
\hline $1823-24$ & 9.035 \\
\hline $1824-25$ & 12.434 \\
\hline $1825-26$ & 9.373 \\
\hline $1826-27$ & 12.231 \\
\hline $1830-31$ & 18.956 \\
\hline $1835-36$ & 30.202 \\
\hline $1838-39$ & 40.200 \\
\hline
\end{tabular}

Sin entrar en detallar las motivaciones que explican la adopción del consumo de opio en la China del siglo XIX, amén de las sociedades del Sudeste asiático, sí mencionaremos varias cuestiones a tener en cuenta. Por el lado de la oferta, fueron determinantes la consolidación de la producción de las factorías de la EIC en la India-que ponía en el mercado un producto de calidad estable, perfectamente adecuado al exigente paladar chino-, además del crecimiento de existencias fruto de las maniobras de la EIC para frenar la seria competencia procedente de las variedades occidentales y muy especialmente, del Malwa, fuera del control británico. Por el lado de la demanda, la cultura del consumo ostentoso en China, junto con la moda por los productos extranjeros (yanghuo re) y la etiqueta y cortesía chinas

3 Elaborado a partir de GREENBERG (1951, p 211), DERMIGNY (1964, III, p. 1266) y TROCKI (1999, p. 95). El total de cajones incluyen las dos variedades de Bengala (Patna y Benarés), el de Malwa y el turco. Un cajón contiene, de media, unos $60 \mathrm{~kg}$ de opio. 
-que apostaban por un producto de gusto refinado, que, contrariamente al consumo de alcohol, no perturbaba el orden social, al no dar lugar a un desenfreno- también tuvieron un peso importante. El papel del opio en las sociedades asiáticas ha sido objeto de profundas revisiones en años recientes, aportando una nueva comprensión que debemos tener en cuenta para comprender el significado del comercio analizado aquí. Dichas revisiones ofrecen un análisis desproblematizado, desprovisto de los juicios de valor propios de la contemporaneidad, aportando unas cifras moderadas de consumo, y relativizando sus efectos. Más que ver en el consumo del opio una "plaga", un síntoma de una sociedad consumida por la adicción y el desgaste moral, a la que las potencias imperialistas habrían "doblegado" contra su voluntad, estos estudios apuntan a unas sociedades asiáticas que habrían adquirido el hábito de un modo más activo, adoptándolo como elemento propio (NEWMAN, 1995, HOWARD, 1998, BROOK - WAKABAYASHI, 2000, MCMAHON, 2002, DIKÖTTER ET AL., 2004, ZHENG, 2005, BELLO, 2005, PAULÈS, 2011). ${ }^{4}$

\section{Redes españolas en el comercio del opio}

La mayor parte del comercio del opio era llevado a cabo entre la India y China a bordo de buques privados, el denominado country trade, dominado en gran parte por súbditos británicos. Aun así, en dicho comercio participaron también otros grupos, que poco a poco van siendo objeto de análisis en la literatura especializada. La tesis doctoral aquí presentada contribuye con el análisis de uno de dichos grupos, con sus condicionantes específicos, en el período que va desde el fin del Galeón de Manila, hasta el inicio del ascenso de las grandes firmas anglosajonas en el sur de China en la década de 1830. En Cantón, firmas como Lorenzo Calvo y $\mathrm{C}^{a}$, así como Yrisarri y $\mathrm{C}^{a}$, durante la década de 1820, recibían consignaciones de opio procedente de varios individuos y corporaciones de la India, entre las que destacaba la también española Manuel Larruleta y $C^{a}$, que en 1823 pasaría a denominarse Mendieta, Uriarte y $C^{a}$. Todas ellas estaban formadas por empleados de la RCF, y en gran parte eran de origen vasco

4 Al margen de la relativización de la gravedad de los efectos y de la adopción activa por parte de las sociedades asiáticas, el opio no deja de ser un instrumento central del imperialismo europeo, muy especialmente por su íntima relación con la viabilidad de las administraciones coloniales, junto con la consolidación de las estructuras capitalistas, como demuestra claramente TROCKI (1999). 
o vasco-navarro. ${ }^{5}$ En China, dichas casas interactuaban con los comerciantes chinos y sus homólogas europeas, muy especialmente las británicas, y se encargaban de vender el opio y mandar las ganancias de vuelta, tras retener honorarios. La plata obtenida se invertía o bien en letras -especialmente de la $\mathrm{EIC}-$, o bien en retornos, tanto en plata en lingotes como azúcar, salitre, canela de China, metales y azufre, además de tejidos de seda y algodón, así como seda en bruto, que se exportaban tanto para la India como para Filipinas.

Las firmas españolas del opio surgieron de las dos factorías de la RCF en Calcuta y Cantón, en un momento de crisis de la corporación, previa a su cese de actividades. A su creación en 1785, la Compañía se dedicó al fomento de las producciones filipinas, para centrarse en el comercio directo entre los puertos asiáticos y americanos desde los últimos años del siglo $\mathrm{XVIII.} \mathrm{Su} \mathrm{balance} \mathrm{fue} \mathrm{positivo} \mathrm{durante} \mathrm{los} \mathrm{primeros} \mathrm{años,} \mathrm{si} \mathrm{bien} \mathrm{las} \mathrm{pérdi-}$ das fueron la norma a partir del cambio de siglo, debido a los conflictos europeos y la independencia de la América hispánica (DÍAZ-TRECHUELO, 1965, pp. 91-153). Como el resto de corporaciones privilegiadas europeas, la RCF permitía un segmento privado de comercio a sus empleados, del que en ocasiones podía sacar partido, usando la liquidez ofrecida por éstos: durante los años aquí analizados, hallamos registros coherentes de una participación intensa en estos negocios privados en Asia y, muy especialmente, en el comercio del opio, entonces en pleno proceso de expansión. Hay, además, indicios puntuales de una participación previa en dicho country trade, y más concretamente, en el tráfico del opio. ${ }^{6}$

La tesis identifica dos entramados españoles en el comercio del opio en Asia oriental, surgidos respectivamente de las factorías de Calcuta y Cantón de la RCF. En la primera, el factor Manuel Larruleta se retiraba de su puesto para fundar la sociedad Manuel Larruleta y $\mathrm{C}^{a}$ en 1818 , junto con otros empleados de la RCF, los jóvenes José de Mendieta y José María Uriarte. Larruleta, por motivos que no trascienden en la documentación utilizada, abandonó la sociedad en 1823, cuando cambió su nombre a Mendieta, Uriarte y $\mathrm{C}^{a}$, e ingresó como socio en la firma británica de Mackintosh \& Co. En junio de 1821, el entramado establece una filial en China, casa con-

5 En cuanto a las redes globales vascas, véase ARAGÓN RUANO, ANGULO MORALES (2013), LAMIKIZ (2010); en cuanto a los vascos en Filipinas, DE BORJA (2005).

6 Acta de la Junta de Gobierno de la RCF, 13 de diciembre de 1811 y 21 de enero de 1812, Archivo General de Indias (en adelante, $A G I$ ), Ultramar, 640; Balance de la RCF del periodo 1805-13, 30 de marzo de 1815, AGI, Filipinas, 993. 
signataria de aquélla, Yrisarri y $C^{a}$, formada por el también empleado de la RCF, Francisco Xavier de Yrisarri, y James Matheson, futuro socio principal de la destacada firma británica de Jardine, Matheson \& Co. ${ }^{7}$

El otro entramado lo formaba la factoría de Cantón, con el factor Lorenzo Calvo y Mateo al frente, un destacado militante de la causa liberal, a quien el gobierno de Fernando VII puso precio a su cabeza en 1830. Calvo, que inició sus actividades privadas sin haber formado sociedad, y quien actuaba con una sólida red de contactos desde Filipinas, dominó el comercio del opio cantonés por lo menos entre 1821 y 22 . A finales de 1822, y después el cierre de la factoría de la RCF en China en 1821, abandonó China, tras haber fundado la sociedad Lorenzo Calvo y $\mathrm{C}^{a}$, junto con Gabriel de Yruretagoyena. Instalado en París por lo menos desde 1827, estableció una red de sociedades entre Asia y Europa -Laurent Calvo et $\mathrm{C}^{i e}$ en la capital francesa, Bergmiller, Calvo et $\mathrm{C}^{\mathrm{ie}}$ en El Havre, además de Calvo, Azcárraga y $\mathrm{C}^{a}$ y Calvo, Roxas y $\mathrm{C}^{a}$, ambas en Manila-, dedicada al comercio transcontinental de productos de plantación filipinos, tejidos chinos, manufacturas europeas varias, además de concentrarse en las inversiones en el sector secundario filipino -junto con destacados emprendedores como José de Azcárraga y Domingo Roxas-, además de mantenerse en las inversiones del opio en China y el comercio intraasiático. El joven Eugenio de Otadui entraba en la sociedad de China en $1827 .{ }^{8}$

La tesis identifica tres fases distintas en la evolución del comercio español del opio. La primera de las etapas se corresponde con los años finales de la década de 1810, coincidiendo con las actividades iniciales de los fac-

7 Balance de la RCF de 1818, 31 de diciembre de 1819, AGI, Filipinas, 994; Acta de Junta de gobierno de la RCF de 12 de agosto de 1825, Filipinas, 983; Yrisarri y $C^{a}$ a Larruleta y $C^{a}$, Cantón-Calcuta, 19 de abril de 1823; a Mendieta, Uriarte y $C^{a}$, Cantón-Calcuta, 26 de abril y 29 de julio de 1823; Jardine Matheson Archive (en adelante, JMA) C2/2; Yrisarri y $\mathrm{C}^{a}$ a Mendieta, Uriarte y $\mathrm{C}^{a}$, Cantón-Calcuta, 3 de octubre de 1825; C2/4; Circular de establecimiento y escritura de constitución de Yrisarri y $C^{a}, 1^{\circ}$ de julio y 10 de octubre de 1821, L11/1 y F10/1; James Matheson a José Antonio Fernández, Cantón-San Sebastián, 15 de enero de $1827, \mathrm{C} 2 / 5$.

8 Yrisarri y $C^{a}$ a Larruleta y $C^{a}$, Cantón-Calcuta, 10 de abril de 1822; a Lorenzo Calvo, Cantón, 27 de octubre de 1822, JMA C2/1; Magniac \& Co. a Calvo, Azcárraga y C ${ }^{a}$, Cantón-Manila, $1^{\circ}$ de octubre de 1828, C10/9; Calvo y $\mathrm{C}^{a}$ a Magniac \& Co., Cantón, 18 de abril de 1827, B7/2 núm. 112; Bottin du Commerce, Archives de Paris PER 292, 1828 a 1833; Acta de sociedad de Bergmiller, Calvo et $C^{i e}$, París, $1^{\circ}$ de marzo de 1828, Archives de Paris D31 U3 36 (2 de enero-10 de mayo de 1828); Acuerdo de Calvo y Roxas, Archivo Histórico de Protocolos Notariales de Madrid, núm. 25559 (agradezco a Martín Rodrigo Alharilla esta última referencia). 
tores Larruleta y Calvo, activos en el clima de reorganización de las redes comerciales europeas en Asia tras el fin del Galeón, y de la que conocemos pocos detalles debido a las pocas fuentes halladas. La segunda se inicia en 1821, con la creación de la sociedad de Yrisarri y $C^{a}$ en Cantón, y de la que sabemos más detalles al conservarse la correspondencia saliente de dicha casa en el Jardine Matheson Archive, en Cambridge (Reino Unido). Esta segunda etapa se desarrolla en plena expansión del sector del opio, que conllevó un aumento de la especulación y la competencia, además de las campañas oficiales chinas contra la sustancia, elementos que acabaron motivando el estancamiento del mercado cantonés. Las firmas españolas, junto con las británicas, elaboraron nuevas estrategias, y exploraron nuevos mercados en Macao, Manila y la costa de Fujian, sacando partido del pabellón español, que tenía acceso a los tres puntos (QUIASON 1966a; CHANG 1983, pp. 266-267; FU, I, pp. 170, 182-183; NG 1983, pp. 55 59). La tercera de las etapas tiene lugar desde 1825 hasta el fin de dichos entramados -en 1827 el de Calcuta, y en 1830 el de Cantón-, y se caracteriza por una relativa estabilización del sector del opio, además de una diversificación de las casas de China, que del mero comercio consignatario pasaron a llevar a cabo iniciativas por cuenta propia, además de tener lugar un mayor nivel de colaboración. ${ }^{9}$ La mayor interdependencia resultante, junto con la fuerte deuda de la RCF contra sus empleados, generó una mayor vulnerabilidad, y finalmente, las firmas españolas no resistieron la crisis financiera del comercio europeo en Asia durante la segunda mitad de la década (CHEONG, 1973). ${ }^{10}$

En conjunto, no es sencillo ofrecer una cifra total de la implicación española durante estos años en el comercio del opio, debido a la parcialidad y dispersión de datos, sin que se hayan podido localizar balances de conjun-

9 De este modo, por ejemplo, Yrisarri y $\mathrm{C}^{a}$ aumentaron su número de corresponsales, con veinticuatro en Calcuta, cinco en Bombay, seis en Singapur, cuatro en Batavia, uno en Penang y otro en Pondicherry; Circular de Yrisarri y $C^{a}$ de 5 de junio de 1824; JMA C2/3.

10 James Matheson a Mendieta, Uriarte y $C^{a}$, Cantón-Calcuta, 3 de marzo de 1827; a José Antonio Fernández, Calcuta-San Sebastián, 4 de junio de 1827; a José de Azcárraga, Cantón-Manila, 26 de septiembre de 1827; JMA C2/5; Gabriel de Yruretagoyena y Eugenio de Otadui a Lorenzo Calvo, Macao-sin determinar, 27 de mayo de 1830, Newberry Library, Ayer MS 1932; Informe de Lorenzo Calvo sobre la RCF de 5 de agosto de 1828 y expediente sobre el asunto Baudin, Etesse et $\mathrm{C}^{\mathrm{ie}}, 9$ de septiembre de 1828, ambos en AGI Filipinas, 996; Expediente sobre el embargo de bienes de Lorenzo Calvo, 4 de enero de 1832, AGI Filipinas, 520; CALVO Y MATEO (1835), 
to de ninguna de las casas analizadas. Como se ha ido diciendo, además, las actividades españolas se llevaron a cabo conjuntamente con individuos de otros orígenes y muy especialmente, británicos, lo que dificulta la identificación de una porción propiamente española dentro de este comercio. Aun así, podemos aventurar que entre 1822 y 1822, alrededor de un $20 \%$ del comercio del opio en el sur de China pasaba por las manos de Yrisarri y $C^{a}$.

\section{Fuentes y estado de La cuestión}

En el estudio del comercio europeo en China han prevalecido unas fuentes en concreto, junto con unas prioridades a la hora de abordarlas. Hasta fechas relativamente recientes, ha habido un énfasis en lo anglosajón, tanto en fuentes como en relatos -si bien este elemento no deja de ser de suma importancia-, obviando así otros grupos, con sus condicionantes específicos que además, fueron determinantes para el desarrollo de las actividades de británicos y estadounidenses. Esta limitación se debe, en primer lugar, a las prioridades establecidas por el modelo historiográfico del "sistema de Cantón", nombre con el que se ha pretendido destacar la especificidad de las complejas particularidades del comercio que tenía lugar en dicha ciudad desde principios del siglo XVIII hasta 1842 (GREENBERG, 1951, DERMIGNY, 1964). Hasta fechas recientes, este modelo ha tendido a segmentar por estructuras nacionales las actividades de las distintas comunidades que se agrupaban en el sur de China, obviando los elementos de colaboración. La atención se ha puesto, además, en unas dinámicas específicas de conflicto, que llevaron a la primera guerra del opio, y en unas rutas en concreto, que unían la India con China, obviando otras rutas de la región. ${ }^{12}$ Esto afecta, en concreto, a la relevancia de las redes del Galeón en el comercio exterior chino y a su sistema productivo. En segundo lugar, el olvido de los españoles en el comercio cantonés se debe a la separación

11 Cifras calculadas a partir de la correspondencia saliente de Yrisarri y $C^{a}, J M A, C 2 / 1$ a 5 , cotejado con las cifras totales de TROCKI (1999, p 95). Basado también en las letras de la EIC adquiridas por Yrisarri y $\mathrm{C}^{a}$ en el periodo indicado (CHEONG, 1979, p 101).

12 El comercio cantonés, junto con la actitud china hacia el mar, está siendo objeto de revisión, con VAN DYKE $(2005,2011)$ a la cabeza. Destacamos también HAO (1986), CHEONG (1997), ZHENG (2011) y ZHAO (2013); véase también la tesis de máster de KUBLER (2014) (agradezco sinceramente al autor el haber compartido amablemente conmigo sus resultados). Hay revisiones más específicas, acerca del comercio holandés del té en Cantón (LIU, 2007), el comercio estadounidense en China (DOWNs, 1968, 1997, GRANT, 1987, 1988, 2012, 2014, VAN DYKE, 2012), parsi (PALSETIA, 2008), o portugués (GUIMARÃES, 1996). 
entre los historiadores del comercio exterior chino y sus homólogos respecto a Filipinas y el comercio del Galeón. La falta de sensibilidad hacia los flujos del comercio global en ambos campos ha motivado el olvido de las importantes conexiones hispánicas con China y la región asiática, salvo algunas excepciones. ${ }^{13}$

El Jardine Matheson Archive ha sido una de las principales fuentes utilizadas para el presente estudio. Siendo una fuente de información fundamental acerca del comercio cantonés, este archivo, que conserva la documentación de la que fue la principal firma británica en el sector del opio, Jardine, Matheson \& Co., ha sido abordado aquí replanteando el análisis al uso, situando las actividades de la firma en un contexto más amplio, más allá de la segmentación por estructuras imperiales del comercio europeo en Asia, y observando, por ejemplo, la abundante documentación relativa a Filipinas y el mundo hispánico: en concreto, este archivo conserva la correspondencia de Yrisarri y $\mathrm{C}^{a} .{ }^{14}$ Una aproximación similar debería hacerse en otra de las fuentes fundamentales en el comercio cantonés, es decir, la documentación de la EIC, que se conserva en el India Office Records, en la British Library, Londres, una de las más extensas y coherentes al respecto, que ha dado lugar, además, a uno de los principales manuales acerca del comercio europeo en china, la extensa obra de MORSE (1926). ${ }^{15}$

Junto con el Jardine Matheson Archive, el Archivo General de Indias, en Sevilla, ha sido la otra fuente principal utilizada en este trabajo, para dilucidar la relación de la RCF con los negocios privados de sus empleados y en concreto, del opio. Dicho archivo, sin embargo, conserva documentación no propiamente de la Compañía sino de la administración borbónica acerca de aquélla, que rara vez aporta el pormenor de las actividades en las factorías o las relaciones entre éstas. Esto se ha tratado de compensar con los diarios del que fue primer factor en China de dicha corporación

13 Debe así tenerse en cuenta CHEONG (1965a), FRADERA (1999a, 2005), ALONSO ÁlVAREZ (2004), MARTÍNEZ SHAW (2007), MARTÍNEZ SHAW - ALFONSO MOLA (2007), IRIGOIN (2009).

14 Acerca de Jardine, Matheson \& Co., véanse GREENBERG (1951), CHEONG (1979), LE PICHON $(1998,2006)$.

15 Puntualmente, se ha cotejado la obra de MORSE (1926), ya mencionada, acerca de la EIC, para hallar detalles acerca de las relaciones de la Honourable Company tanto con la RCF como con los negocios privados de sus empleados. En trabajos futuros analizaré la documentación de la EIC para aportar más detalles acerca de la colaboración hispano-británica. 
a finales del siglo XVIII, Manuel de Agote, y que se conservan en el Untzi Museoa-Museo Naval de San Sebastián. ${ }^{16}$ Asimismo, se han utilizado el ya desaparecido Archivo del Ministerio de Asuntos Exteriores de Madrid, el Archivo Histórico Nacional, el Archivo de la Real Academia de la Historia así como los National Archives of the Philippines, cuyos fondos españoles están microfilmados en el Instituto de Historia del Consejo Superior de Investigaciones Científicas, en Madrid; los intereses de Calvo en Europa, además, han sido reseguidos en el Archivo municipal de París y los Archivos nacionales franceses.

Como hemos dicho, en la selección de una fuentes en concreto y de las cuestiones que se buscan en ellos, los españoles han sido olvidados en los estudios del comercio exterior chino y, si aparecen, lo hacen de un modo tangencial, más como excepción, tratando de explicar qué hacían en un orden imperial británico. No han sido analizados con sus condicionantes específicos ni complejidades internas, ni su posición dentro de un imperio español, con sus ramificaciones en Asia, entonces en pleno proceso de reconfiguración en dicha región ante la independencia de América Latina. Una excepción a esto son los distintos trabajos de W. E. Cheong, que observa la falta de aproximación a las redes hispánicas en el comercio cantonés, así como muestra una comprensión de cómo los procesos de cambio en la región entre finales del siglo XVIII y principios del XIX afectaron profundamente al papel de la economía filipina y sus conexiones regionales (CHEONG, 1965a, 1965b, 1970, 1971, 1973, 1979). Otra importante excepción es la de FRADERA (1999b), quien aporta direcciones metodológicas acerca de la participación española en el comercio del opio en su análisis del factor Calvo. ${ }^{17}$

\section{TRANSICIONES Y CAMBIOS}

El protagonismo español en el comercio del opio ilustra los complejos procesos de transición regional y global que afectaron al comercio europeo en Asia durante las primeras décadas del siglo XIX. En medio de tales procesos de cambio, la presencia española fue corta en el tiempo, lo que

16 En este sentido, el tan citado trabajo de DÍAZ-TRECHUELO (1965) necesita una profunda revisión, con el aporte de nuevas fuentes, que analicen la RCF en un contexto mucho más amplio, más allá de los intereses meramente metropolitanos reflejados en la documentación del Archivo General de Indias.

17 Más recientemente, Alain Le Pichon recoge las observaciones de Cheong en sus obras (LE PICHON, 1998, 2006). 
también explicaría tal vez su ausencia en el discurso historiográfico. Los estudios sobre el comercio cantonés tienden a pasar por alto este periodo, centrándose más en el siglo XVIII o en los años inmediatamente anteriores a la primera guerra del opio, además de dejar de lado los procesos que tenían lugar en los sistemas económicos que afectaban al comercio en el sur de China. Las actividades de los españoles deben situarse en este contexto de cambio e incertidumbre, al ofrecer estos bazas ventajosas en un momento sin alternativas claras a corto plazo; a dicho ciclo de cambio se debe, también, su desaparición.

El fin del ciclo revolucionario europeo conllevó la consolidación de la posición británica en el comercio asiático. Aun así, ésta no era del todo definitiva hasta los años inmediatamente anteriores a la primera guerra del opio, y paralelamente a las mayores esferas de monopolio del comercio británico en Asia oriental, había todavía otras iniciativas que prosperaban junto con aquél, incluso colaborando intensamente. Hasta el alto imperialismo de mediados del siglo XIX, además, no se había consolidado la segregación de carácter nacional -por no decir, racial- del mundo de los negocios en el contexto colonial. ${ }^{18}$ Dicho ascenso británico se iba haciendo a expensas, paulatinamente, del monopolio de la EIC, al mismo tiempo que aumentaba la navegación privada británica en la región-gracias a la Charter Act de 1813, que entre otras medidas, abría los puertos indios a la libre iniciativa, o a la apertura del puerto libre de Singapur en 1819-. De este modo, la iniciativa privada prosperó en las parcelas permitidas por la Compañía que todavía mantuvo el monopolio en el comercio británico en China hasta 1834-, y muy especialmente, en las ventas en China y el Sudeste asiático del opio adquirido en la India, cuya distribución era delegada así a terceros por la EIC, por no querer entrar en conflicto con las autoridades chinas a raíz de su política anti-opio (BAYLY, 1989).

La afectación del ciclo revolucionario en el imperio español tuvo también una importante repercusión en Asia. La finalización del Galeón en 1815, en las cortes de Cádiz, consolidó el paso definitivo en Filipinas de una economía de redistribución de importaciones a la producción de productos de plantación para su exportación, proceso ya iniciado desde el último tercio del siglo XVIII (FRADERA, 2005, pp. 475-486) lo que hacía aumentar los intereses filipinos en la región. No en vano, en 1814 Manila se abría definitivamente al comercio internacional, tras haberse implementado gradualmente desde 1789 (MARTíNEZ SHAW, 2007, pp. 52-70). Durante los años inmediatos al fin del Galeón, hubo ciertas iniciativas en el comercio 
privado, tanto con el Pacífico mexicano como con la región asiática, lo que atañe también a las firmas privadas españolas en Cantón y Calcuta (CHEONG, 1971, VALDÉS LAKOWSKY, 1987, LEGARDA, 1999, 2002, PÉREZ LECHA, 2014). El estatuto de la RCF durante las primeras décadas del siglo XIX también era poco claro, debido a la puesta en entredicho de su privilegio tanto por las cortes gaditanas como por las diversas medidas liberalizadoras en lo económico que prosiguieron aun después del Trienio Liberal. Esto afectó a sus ya maltrechas actividades.

Huelga decir que la independencia de la América hispánica hacia 1820 afectó de lleno al comercio asiático, muy especialmente en cuanto a la distribución de la plata americana se refiere. Su escasez, junto con su apreciación, generó un descalabro económico en la China de las eras Jiaqing (1796-1820) y Daoguang (1820-50), agravando el proceso de crisis social y declive dinástico, alimentando además los recelos Qing hacia el opio, al que se responsabilizaba de ser el principal motivo del "hambre de plata" (yinhuang). ${ }^{19}$ Junto con las campañas anti-opio de las primeras décadas del siglo XIX, el gobierno Qing emitía distintos edictos prohibiendo la exportación del metal desde 1809 (MORSE, 1926, III, pp. 127-129). Al mismo tiempo, la escasez de numerario, afectó de lleno al comercio cantonés, especialmente entre los años 1812-15.

Los procesos de independencia en América reconfiguraron también la posición española en Asia. Por un lado, las iniciativas privadas transpacíficas recién mencionadas quedaron afectadas, a las que la expulsión definitiva de los españoles de México en 1827 acabaron de dar el toque de gracia. Por el otro, la suerte de la RCF quedaba sentenciada, al quedarse sin la parte más significativa de su negocio (DÍAZ-TRECHUELO, 1965, pp. 119-153, LEGARDA, 1999, pp. 80-83). No en vano, la factoría de China se cerraba en 1821, al mismo tiempo que hubo esfuerzos en desarrollar el comercio indio con la Península. Éstos, sin embargo, no prosperaron: con el fin de la firma de Mendieta, Uriarte y $\mathrm{C}^{a}$ en 1827, se extinguían las actividades de la factoría española en Calcuta. Tras la revolución liberal, el privilegio de la RCF finalizaba en 1834, dando lugar a un largo proceso de liquidación.

En conjunto, estos procesos de transición afectaron también a la naturaleza del comercio privado europeo en Asia. Durante los años 1820 prospe-

19 Esto ha sido puesto en tela de juicio en fechas recientes; LIN (2007). Los trabajos de Alejandra Irigoin han señalado certeramente que con la independencia de Hispanoamérica no finalizó el suministro de plata en sí, sino que desapareció el peso fuerte español, divisa fuerte que tenía una gran demanda en los mercados asiáticos (IRIGOIN, 2009). 
raron las iniciativas privadas, bajo el paraguas protector del privilegio de los sistemas monopolísticos, entonces en proceso de declive. Tras el fin de éstos, y con el auge del libre comercio, el sector estará paradójicamente dominado por menos firmas, con medios posibles para prosperar, limitando de este modo las capacidades de las iniciativas de tamaño pequeño y mediano. Dicha situación de coexistencia entre intereses privilegiados y privados se mantuvo en el caso británico hasta el fin efectivo del monopolio de la Compañía en el comercio chino en 1834, mientras que las factorías asiáticas de la RCF desaparecieron en 1821 en el caso de Cantón, y hacia 1827 en la de Calcuta, como hemos visto.

Una mención a parte merecen las transformaciones del propio comercio del opio, objeto no solamente de un crecimiento exponencial, sino de cambios imprevisibles con la irrupción de nuevos actores y condicionantes en él. Con todo, tenemos durante estos años un aumento de la demanda, lo que atrajo a cada vez más especuladores, que no dudaban en imponer prácticas agresivas, lo que, junto con el descontrol del suministro de la EIC ante la competencia del Malwa, daba lugar a graves oscilaciones en el comercio del opio en Cantón y Macao.

Con todo, en este momento de transición y profunda incertidumbre, prosperaron las iniciativas privadas españolas, al colaborar de cerca con los británicos -colaboración que tenía precedentes por lo menos desde que la RCF se estableció en Asia-. Los españoles ofrecían beneficios en un contexto de cambio constante, muy especialmente en cuanto a la escasez de fuentes de numerario se refiere, algo que el factor Calvo supo aprovechar durante los años finales de la década de 1810, cuando las tres casas británicas que dominaban el sector estaban en proceso de reconfiguración, resultado de la crisis de numerario del mercado cantonés entre 1812 y 1815. Inicialmente, entre el fin del Galeón y antes de la independencia de las repúblicas hispanoamericanas, los españoles en China podían ofrecer fuentes alternativas de plata, por lo menos hasta la última expedición de la RCF al Perú en 1819-20 (PARRÓN SALAS, 1995, pp. 402-407), así como hubo expediciones privadas al Pacífico mexicano, como hemos visto. Desde 1820, sin embargo, la principal baza ventajosa de los españoles en China fue su acceso a Filipinas, en un momento en el que el sector del opio precisaba de mayor solidez financiera, como veremos.

El mismo proceso de cambio también explica el fin de las iniciativas españolas en la década de 1830. Sin el apoyo de la RCF, las firmas españolas se encontraban en inferioridad de condiciones, teniendo que competir, además, con grandes corporaciones como Jardine, Matheson \& Co., Dent \& Co. y Russell \& Co., con amplios recursos en personal, apoyo logístico y amplias redes internacionales de financiación. El crédito procedente de Manila, además, se reorientó a otros sectores debido a la consolidación del 
nuevo modelo económico filipino, mientras que en China adquieren mayor relevancia otras fuentes de financiación, muy especialmente procedente de Inglaterra y los Estados Unidos.

\section{LA COLABORACIÓN HISPANO-BRITÁNICA}

Las actividades españolas en el tráfico del opio se desarrollaron en estrecha cooperación con los comerciantes británicos, ejemplificando así el carácter colaborativo del comercio cantonés, menos segregado por segmentos nacionales como hasta recientemente se ha tendido a caracterizar. Dicho carácter colaborativo tuvo lugar entre todos los actores implicados, entre comerciantes europeos - de distintos países, tanto de compañías privilegiadas como privadas- como chinos -janistas, comerciantes exteriores-, además de los distintos rangos de la burocracia china, más abiertos al comercio internacional de lo que se ha tendido a percibir. Como demuestra el conjunto de trabajos de Paul van Dyke, la colaboración, la armonía y el bien común eran objetivos deseables por todos los integrantes del comercio cantonés, y en ocasiones, había efectivamente conflictos.

Los dos entramados surgidos de las dos factorías de la RCF implicadas en el comercio del opio interactuaron estrechamente con intereses británicos. La principal firma británica del opio durante los años 1820, Charles Magniac \& Co. - precedente directo de Jardine, Matheson \& Co.-, contrató en repetidas ocasiones los servicios del factor Calvo para el almacenaje, distribución y venta del opio en aguas de Macao, usando el pabellón español -el único europeo permitido, al margen del portugués-. Calvo actuaría, además, como apoderado en China de las firmas filipinas ante las casas británicas en Cantón, algo que sus predecesores en la factoría ya habían hecho. Tras catorce años como empleado de la RCF en Manila, a su llegada en China en 1816 traería consigo una extensa red de contactos que todavía está pendiente de conocerse mejor. Tanto sus prácticas como su captación de redes filipinas serían imitadas por Yrisarri y $\mathrm{C}^{a}$.

En el entramado de Calcuta, las complicidades hispanobritánicas eran tal vez más estrechas. Desde que el factor Larruleta creara la sociedad que lleva su nombre, sus actividades estarán íntimamente relacionadas con la firma de Mackintosh \& Co., de Calcuta, una de las grandes casas del índigo, que también estaba plenamente implicada en la adquisición del opio en las subastas de Calcuta para su consignación para China. Cuando Larruleta se retire de su sociedad, ingresará como socio en dicha firma, que además, era suministradora de la RCF. Cuando finalmente, en 1833, Mackintosh \& Co. se declare en suspensión de pagos, Mendieta, Uriarte y $\mathrm{C}^{a}$, que había quebrado en 1827 , será su segunda deudora. Por otro lado, la filial de China del entramado de Calcuta, Yrisarri y $C^{a}$, estaba formada por el vasco-navarro Francisco Xavier de Yrisarri y el escocés James Mathe- 
son, que además, pertenecía al entorno familiar de los Mackintosh, lo que claramente buscaba acercar los intereses británicos con las redes filipinas; no en vano, la elección de un nombre hispánico para la nueva sociedad estaba seguramente orientado a la captación de la potencial clientela en el archipiélago. Yrisarri y $C^{a}$ tenían, además, clientela muy diversa entre los consignadores de la India, y hay indicios de que algo parecido sucedía con la firma de Calvo en Cantón. Junto con las principales corresponsales -los Mackintosh, Larruleta, Mendieta y Uriarte-, aparecen en la correspondencia de la firma hispanobritánica muchos nombres ingleses e indios. Yrisarri, además, había sido formado en el mundo comercial británico, como gran parte de los empleados de la RCF de su generación, y la Compañía tenía no pocos intereses en Inglaterra que todavía deberían ser conocidos.

Las complicidades de los empleados de la RCF con los británicos ya procedían de la época en la que ésta empezó sus actividades en 1785. El interés por la plata y por el comercio filipino explican dicha colaboración: varios son los préstamos concedidos por la compañía española a su homóloga británica, y desde 1796, además, la RCF establecía factoría en Calcuta. Los contratos con la RCF resultaban un modo de evitar los problemas del country trade clandestino que tenía lugar entre Madrás y Manila desde el siglo XVII, comercio que era el segundo en importancia de la colonia británica en la India, después del comercio sino-indio. El interés británico por el archipiélago se hizo patente a raíz de la ocupación de Manila durante la Guerra de los Siete Años, o en las propuestas hechas durante las convenciones de Nutka para la cesión de Luzón a los británicos, para poder desarrollar la economía de plantación, muy especialmente para su exportación a China y compensar así el déficit comercial en Cantón. Durante las guerras napoleónicas, además, los contratos con los españoles de Calcuta eran una solución a la prohibición que pesaba sobre los súbditos británicos de comerciar con las potencias enemigas (FURBER, 1935, 1948, CHAUNU, 1960, CHEONG, 1965b, 1970, 1971, QUIASON, 1966b, TRACY, 1995).

Jardine, Matheson \& Co. será heredera, además, de las complicidades hispano-británicas. La firma era la continuadora de Charles Magniac \& Co., que de ella adquiría los intereses del entramado de Calvo, muy especialmente en la figura de sus agentes en Manila entre 1834 y 1846 , Eugenio de Otadui y $C^{a}$, cuyo socio principal era un antiguo empleado de Calvo (TARLING, 1963). Con el ingreso de James Matheson y su sobrino Alexander en 1827, la firma adquiría sus contactos filipinos, de los que seguiría captando el crédito hasta poco antes de la primera guerra del opio, así como los contactos en la costa pacífica mexicana, como vamos a ver. ${ }^{20}$

20 Incluso heredó contactos con N. M. Rothschild \& Sons, puesto que uno de los socios de Mendieta, Uriarte y $C^{a}$, José Xavier de Barcáiztegui, trabajó para Daniel Weisweller, agente de dicha banca en España. Ésta cobraba sus préstamos al estado español en giros sobre las 


\section{El PROTAGONISMO FILIPINO}

La integración de Filipinas en la economía regional se ha tendido a olvidar en los análisis del comercio cantonés, lo que atañe también al comercio del opio. Además de ser el territorio bajo soberanía europea más cercano a la costa china desde finales del siglo XVI -exceptuando el pequeño enclave sinoportugués de Macao-, debe tenerse en cuenta su conexión con el Pacífico, el comercio del Galeón, sus exportaciones, la acción de la RCF, así como los intereses estadounidenses y británicos en Manila, elementos todos ellos que tendrán su nada menospreciable rol en el comercio cantonés. El proceso de transformación económica, empezado tras la ocupación británica durante la Guerra de los Siete Años, seguramente expandió los productos de plantación por la región, algo que todavía requeriría de un mayor estudio; por lo menos, hay indicios de un aumento de la navegación española en Asia desde el último tercio del siglo XVIII (CHAUNU, 1960). Sabemos que desde 1790, la factoría de la RCF en China acepta las consignaciones mandadas por los comerciantes privados manileños, lo que daría fe de dicha expansión, el mismo año en que Manila empezaba a abrirse al comercio internacional (DÍAZ-TRECHUELO, 1965, p. 78). Todavía precisarían de un mayor estudio las inversiones en préstamos a riesgo de mar puestos en buques que navegaban por la región, al margen de los del Galeón -y que tomaría una nueva dimensión tras el fin de éste y el aumento del comercio del opio-, así como las inversiones españolas en los tesoros de Cantón y Bengala de la EIC, indicios que apuntan también a dicha integración (MORSE, 1926, IV, pp. 189-190, 258-259).

Dicho grado de integración se hará patente con el ascenso del comercio del opio cantonés, como analiza con mayor detalle el estudio aquí presentado. Como hemos indicado, las conexiones de las casas españolas de China con Filipinas fueron su principal ventaja comparativa ante los comerciantes británicos. Esto debe decirse contrariamente a lo que se suele dar por sentado en parte de la literatura sobre el comercio del opio en Cantón, que asume que la conexión directa de los españoles con América y la plata fue su principal ventaja (DERMIGNY, 1964, III, p 1244, CHEONG, 1979, p 51, LIN, 2007, p 110). Si bien dicha conexión habría sido ciertamente relevante durante los primeros años del boom del opio -hemos visto las expediciones al Perú así como al Pacífico mexicano alrededor de 1820-, esta afirmación ignora el profundo proceso de transformación por el que pasaba el imperio

cajas ultramarinas, entre ellas, las de Filipinas, de cuyo cobro se encargaban los agentes de Jardine, Matheson \& Co. en Manila (DE OTAZU, 1987, LÓPEZ-MORELL, 2005, RUIZ DE GORDEJUELA, 2006). 
español en aquel momento, lo que afectó de lleno a el comercio cantonés y a la posición de los súbditos españoles, haciendo problemático su acceso a América y a la plata desde 1820. De este modo, entre 1822 y $1825-$ momento del que conservamos mayor evidencia documental--, los negocios transpacíficos de las casas de China no fueron relevantes, mientras que sus esfuerzos en reforzar la financiación filipina fueron mayores. ${ }^{21}$ En un momento en el que las relaciones de las firmas criollas filipinas con las redes europeas del comercio y las finanzas eran tenues, de reorganización y pleno dinamismo tras el desmantelamiento del Galeón, y en el que las firmas extranjeras todavía no habían consolidado su posición en Manila, los contactos con la colonia eran valorados, lo que los comerciantes británicos explotaron tanto en Cantón y Macao como en Calcuta. Dicho proceso tenía lugar en un momento en el que, además, las élites criollas de la colonia luchaban por afianzar su posición, más a raíz de la implantación de la Constitución de Cádiz (DE LLOBET, 2011).

El negocio del opio precisaba cada vez más de un mayor crédito, para así poder hacer frente a los riesgos resultantes de una competencia feroz, las prácticas especulativas y las persecuciones chinas. Mientras que Charles Magniac \& Co. o W. S. Davidson \& Co. recurrían a redes de financiación en los mercados financieros de Estados Unidos e Inglaterra, usando mecanismos de transmisión de capital que justo se empezaban a implementar en aquel momento, otras como Calvo y $\mathrm{C}^{a}$ e Yrisarri y $C^{a}$ usaban de la próxima Filipinas. Después del fin del Galeón, los inversores filipinos buscaban nuevas inversiones: parte del capital fue a las casas españolas de China, que se ponía en fondos de inversión a un alto interés anual de un $20 \%$, para ser utilizados en préstamos a riesgo de mar, cuyos tomadores eran en su mayor parte propietarios de buques macaenses en la navegación asiática. Asimismo, una parte también se ponían en depósitos a un plazo fijo del $10 \%$ anual. De Manila procedieron también importantes préstamos a las casas de China, así como a la firma española de Calcuta, Mendieta, Uriarte y $C^{a}$, amén de financiar otros negocios de Yrisarri y $C^{a}$.

De este modo, nombres como los de Juan de Córdova, Baltasar Mier, Manuel de Revilla, Yñigo González de Azaola, Ventura de Pereda o Dolores de Yruretagoyena -hija del regidor de Manila, José Domingo de Yruretagoyena- destacan entre los principales financiadores. Por ejemplo, en 1825, Pereda, Revilla e Yruretagoyena destacan con 12.000 pesos fuertes,

21 De este modo, Yrisarri y $\mathrm{C}^{a}$ hablan del «semblante poco favorable» del comercio mexicano a un corresponsal manileño en 1822; Yrisarri y $C^{a}$ a Nicolás de Molina, Cantón-Manila, 22 de septiembre de 1822, JMA C2/1. 
18.000 los otros dos, amén de 24.000 pesos fuertes más adelantados por los dos últimos conjuntamente. ${ }^{22}$

Las firmas de China también continuaron con los servicios que la propia factoría de la RCF ofrecía desde 1790, aceptando consignaciones y mandando retornos de vuelta para Manila. Asimismo, ofrecían servicios todavía por desarrollar en la colonia, especialmente de banca y seguros, así como transmitían información financiera de los mercados indio y europeo. Yrisarri y $\mathrm{C}^{a}$ aceptaba consignaciones, por ejemplo, de la firma manilense de Yrastorza, Brodett y $\mathrm{C}^{a}$-uno de cuyos socios, Manuel Brodett, era factor de la RCF en Manila- de arroz, índigo, maderas, cigarros y productos gastronómicos destinados al consumo chino -pepino de mar, nido de salangana, aleta de tiburón-, y organizaban retornos en textiles, azufre, plomo, salitre y a veces, té. Ocasionalmente, Manila también se usará como punto de redistribución del opio cantonés, para su venta a los juncos procedentes de Fujian; de este modo, entre 1823 y 1824, Yrisarri y $C^{a}$ mandarán algunas partidas de opio a José de Azcárraga para su venta, solución que sería utilizada puntualmente en ocasiones posteriores. Años después, ante la campaña del alto comisionado imperial Lin Zexu para erradicar el opio de la provincia de Guangdong, Jardine, Matheson \& Co., junto con otras firmas británicas, estadounidenses y macaenses, enviarán sus existencias a Manila; en el caso de la firma mencionada, sus agentes Eugenio de Otadui y $\mathrm{C}^{a}$ se encargarán de su recepción y venta. ${ }^{23}$

Los intereses de Manila, Cantón y Calcuta también se juntaron en intentos para reemprender el comercio transpacífico, entonces muy afectado por los procesos de independencia. Si bien hemos observado cómo dichos contactos fueron complicados, en 1825 el entramado de Yrisarri y $C^{a}$ y Mendieta, Uriarte y $\mathrm{C}^{a}$ organizarán expediciones a Mazatlán (Sinaloa), aprovechando las medidas favorecedoras del gabinete de Guadalupe Victoria, antes de la expulsión de los españoles de 1827. De este modo, se sentaron las bases del futuro comercio de textiles asiáticos por plata americana que

22 Yrisarri y $C^{a}$ a Mendieta, Uriarte y $C^{a}$, Cantón-Calcuta, 24 de septiembre de 1825, JMA C2/4 Debido al carácter fragmentario de la correspondencia, no se pueden facilitar datos totales de las cantidades puestas desde Manila.

23 Yrisarri y $C^{a}$ a Mendieta, Uriarte y $C^{a}$, Cantón-Calcuta, 26 de abril, 29 de julio y 24 de septiembre de 1823; a José de Azcárraga, Cantón-Manila, 4 de diciembre de 1823; JMA C2/2; Jardine, Matheson \& Co. a Otadui y $\mathrm{C}^{a}$, Cantón-Manila, 11 de marzo de1839; Circular de Jardine, Matheson \& Co., 22 de junio de 1839, C10/25. 
casas como Jardine, Matheson \& Co. continuarían en años posteriores (MCMASTER, 1959, VALDÉS LAKOWSKY, 1987, pp. 293-294, HEATH, 1993). ${ }^{24}$

\section{Conclusión: UNA PRESENCIA No tan Singular}

A modo de conclusión, la tesis doctoral "La participación española en la economía del opio en Asia Oriental tras el fin del Galeón" ilustra una presencia española en el comercio asiático, durante las primeras décadas del siglo XIX, que más que una excepción, debe verse como un elemento establecido desde la llegada de los españoles en Asia durante la "primera globalización". Dicha presencia habría permanecido en Asia, a medida que la posición del resto de potencias habría ido creciendo, habría creado su propia compañía privilegiada en un periodo relativamente tardío -la RCF- y habría participado activamente del comercio del opio, además de implicarse en el country trade regional. Si bien sus números serían relativamente pequeños en relación al resto de potencias europeas -y muy especialmente, la EIC o la Compañía Holandesa de las Indias orientales-, puesto en el conjunto del imperio español, no estaríamos hablando de un comercio tan pequeño. Como hemos venido insinuando, faltan todavía mayores estudios que detallen la integración de Filipinas en la región a raíz de las reformas borbónicas, y asimismo, otros indicios apuntan a una importante implicación de las redes del imperio español en la economía asiática, y muy especialmente, en cuanto a la integración del Galeón y la plata se refiere, cuya producción fue en aumento durante el siglo XVIII. La presencia española en el comercio cantonés también fue más significativa tras la invasión de Manila en 1762-64, y no en vano, el sistema de las Tres Colonias subsistió hasta 1898, debido al relativo vigor de las medidas para afianzar la economía en dichos lugares, incluido Filipinas, y su integración regional.

De este modo, el presente estudio debe verse como una aportación a la necesaria integración del elemento hispánico en los discursos sobre el imperialismo y el comercio en Asia. Con todo, la presencia de súbditos españoles en las redes de comercio de Asia oriental debe verse como muestra de los largos vínculos regionales y globales del comercio cantonés, con interacciones con otros poderes europeos, y en cómo las redes de individuos, más que las estructuras nacionales, tenían más relevancia, elementos que deberán ser confirmados con futuros trabajos.

24 La expedición de la fragata Merope a Mazatlán, con la financiación de Calcuta, Cantón y Manila, a mediados de 1825, con un cargamento de textiles a cambio de plata, fue la más destacada; Yrisarri y $\mathrm{C}^{a}$ a Mendieta, Uriarte y $\mathrm{C}^{a}$, Cantón-Calcuta, 2 de mayo, 14 y 18 de junio de 1825, JMA C2/4. 


\section{Biblografía}

ALONSO ÁlVAREZ, L. (2004): "El impacto de las reformas borbónicas en las redes comerciales. Una visión desde el Pacífico hispano, 1762-1815". II Congreso de la Asociación Mexicana de Historia Económica, UNAM, 27. 29 de octubre de 2004.

ARAGÓN RUANO, Á.; ANGULO MORALES, A. (2013): "The Spanish Basque country in global trade networks in the eighteenth century". International Journal of Maritime History, XXV/1, pp. 149-172.

BAYLY, C. A. (1989): Imperial Meridian. The British Empire and the World. 1780-1830. Londres, Longman.

BELLO, D. A. (2005): Opium and the Limits of Empire. Drug Prohibition in the Chinese Interior, 1729-1850. Cambridge (MA), Harvard University Asia Center. BOUCHON, G. (1991): "Notes on the Opium Trade in Southern Asia during the Pre-Colonial Period", en PTAK, R.; ROTHERMUND, D. (Eds.): Emporia, commodities and entrepreneurs in Asian maritime trade, c. 1400-1750. Stuttgart, Franz Steiner Verlag, pp. 95-106.

BROOK, T.; WAKABAYASHI, B. T. (eds.) (2000): Opium Regimes. China, Britain, and Japan, 1839-1952. Berkeley (CA), University of California Press. CALVO Y MATEO, L. (1835): Esposición presentada a la Junta Liquidadora de la estinguida Real Compañía de Filipinas, por Don Lorenzo Calvo, su Factor que fue en China hasta 1822, reclamando un Crédito á su favor y contra la Compañía de pesos fuertes 542,933:7rs.5g. Madrid, Imprenta de Villaamil.

CHANG P.-t. (1983): Chinese Maritime Trade: The case of Sixteenth-Century Fu-chien (Fukien). Ann Arbor (MI), UMI Dissertation Services.

CHAUNU, P. (1960): Les Philippines et le Pacifique des Ibériques (XVle, XVle, $X V I I l e$ siècles). Introduction méthodologique et indices d'activité. París, SEVPEN.

CHEONG, W. E. (1965a): "Trade and Finance in China: 1784-1834. A Reapp.raisal". Business History, VII, pp. 34-56.

- (1965b): "An Anglo-Spanish-Portuguese Clandestine Trade between the Ports of British India and Manila, 1785-1790". The Philippine Historical Review, I/1, pp. 80-94.

- (1970): "Changing the Rules of the Game (The India-Manila Trade: 17851809)". Journal of Southeast Asian Studies, 1/2, pp. 1-19.

- (1971): "The Decline of Manila as the Spanish Entrepôt in the Far East, 1785-1826: Its Impact on the Pattern of Southeast Asian Trade". Journal of Southeast Asian Studies, 2/2, pp. 142-158.

- (1973): "China Houses and the Bank of England Crisis of 1825". Business History, 15/1, pp. 56-73. 
- (1979): Mandarins and Merchants. Jardine Matheson \& Co., a China agency of the early nineteenth century. London, Curzon Press.

- (1997): Hong Merchants of Canton. Chinese Merchants in Sino-Western Trade, 1684-1798. Richmond (Reino Unido), Curzon Press.

DE BORJA, M. R. (2005): Basques in the Philippines. Reno (NV), University of Nevada Press.

DE LLOBET, R. (2011): "Orphans of Empire: Bourbon Reforms, Constitutional Impasse, and the Rise of Filipino Creole Consciousness in an Age of Revolution". Tesis doctoral, Madison (WI), University of Wisconsin-Madison.

DE OTAZU, A. (1987): Los Rothschild y sus socios en España (1820-1850). Madrid, O. Hs. Ediciones.

DERKS, H. (2012): History of the Opium Problem. The Assault on the East, ca. 1600-1950. Leiden, Brill.

DERMIGNY, L. (1964): La Chine et l'Occident. Le commerce à Canton au XVIII' siècle. 1719-1833. París: SEVPEN.

DÍAZ-TRECHUELO, M. L. (1965): La Real Compañía de Filipinas. Sevilla, Publicaciones de la Escuela de Estudios Hispano-Americanos de Sevilla.

DIKÖTTER, F.; LAAMANN, L.; ZHOU X. (2004): Narcotic Culture. A history of drugs in China. Londres, C. Hurst \& Co. Publishers.

FRADERA, J. M. (1999a): Filipinas, la colonia más peculiar. La hacienda pública en la definición de la política colonial, 1762-1868. Madrid, Consejo Superior de Investigaciones Científicas.

- (1999b): "Opio y negocio, o las desventuras de un español en China", en Gobernar colonias. Barcelona, Península, pp. 129-152.

- (2005): Colonias para después de un imperio. Barcelona, Edicions Bellaterra.

FU, L.-s. (1966): A Documentary chronicle of Sino-Western relations: 16441820. Tucson (AZ), Association for Asian Studies by the University of Arizona Press.

FURBER, H. (1935): "An Abortive Attempt at Anglo-Spanish Commercial Cooperation in the Far East in 1793". The Hispanic American Historical Review, XV/4, pp. 448-463.

- (1948): John Company at Work. A Study of European Expansion in India in the Late Eighteenth Century. Cambridge (MA), Harvard University Press. GRANT, F. D., Jr. (1987): "Hong Merchant Litigation in the American Courts". Proceedings of the Massachusetts Historical Society, XCIX, pp. 44-62.

- (1988): "The Failure of the Li-ch'uan Hong: Litigation as a Hazard of Nineteenth Century Foreign Trade". American Neptune, 48:4, pp. 243-260. 
- (2012): "The April 1820 Debt Settlement between Conseequa and Benjamin Chew Wilcocks", en VAN DYKE, P. A. (Ed.): Americans and Macao. Trade, Smuggling, and Diplomacy on the South China Coast. Hong Kong, Hong Kong University Press.

- (2014): The Chinese Cornerstone of Modern Banking. The Canton Guaranty System and the Origins of Bank Deposit Insurance. 1780-1933. Leiden, Brill. GREENBERG, M. (1951): British Trade and the Opening of China. 1800-42. Cambridge, Cambridge University Press.

GUIMARÃES, Â. (1996): Uma relação especial. Macau e as relações luso-chinesas (1780-1844). Lisboa, Centro de Investigação e Estudos de Sociologia. HAO, Y.-p. (1986): The Commercial Revolution in Nineteenth-Century China. The Rise of Sino-Western Mercantile Capitalism. Berkeley (CA), University of California Press.

HEATH, H. (1993): "British Merchant Houses in Mexico, 1821-1860: Conforming Business Practices and Ethics". The Hispanic American Historical Review, 73/2, pp. 261-290.

HOWARD, P. W. (1998): Opium Supp.ression in Qing China: Responses to a Social Problem, 1729-1906. Ann Arbor (MI), UMI Dissertation Services.

IRIGOIN, A. (2009): "The end of a silver era: the consequences of the breakdown of the Spanish Silver Peso standard in China and the United States, 1780s-1850s". Journal of World History, 20/2, pp. 207-243.

KUBLER, C. E. (2014): "Communication, Cooperation, and Conflict: Rethinking Everyday Qing-Foreigner Relations under the Canton System, 18001839". Trabajo de fin de máster, University of Chicago.

LAMIKIZ, X. (2010): Trade and trust in the Eighteenth-Century Atlantic World. Spanish Merchants and their Overseas Networks. Woodbridge, Suffolk (Reino Unido) y Rochester (NY), Boydell Press.

LEGARDA, B. J., Jr. (1999): After the Galleons. Foreign Trade, Economic Change and Entrepreneurship in the Nineteenth-Century Philippines. Madison (WI), University of Wisconsin-Madison.

- (2002): "Las consecuencias económicas para Filipinas de la independencia de México". Illes i imperis, 6, pp. 125-135.

LE PICHON, A. (1998): Aux origines de Hong Kong. Aspects de la civilisation commerciale à Canton: le fonds de commerce de Jardine, Matheson \& Co. 1827-1839. Paris, L'Harmattan.

- (Ed.) (2006): China Trade and Empire. Jardine, Matheson \& Co. and the Origins of British Rule in Hong Kong, 1827-1843. Oxford, The British Academy by Oxford University Press. 
LIN, M.h. (2007): China Upside Down: Currency, Society, and Ideologies, 1808-1856. Cambridge (MA), Harvard University Asia Center.

LIU Y. (2007): The Dutch East India Company's Tea Trade with China, 17571781. Leiden, Brill.

LÓPEZ-MORELL, M. Á. (2005): La casa Rothschild en España (1812-1941). Madrid, Marcial Pons.

MARTíNEZ SHAW, C. (2007): El sistema comercial español del Pacífico (17651820). Discurso leído el día 11 de noviembre de 2007 en la recepción pública del Excmo. Sr. D. Carlos Martínez Shaw y contestación por la Excma. Sra. D. Carmen Iglesias. Madrid, Real Academia de la Historia.

MARTíNEZ SHAW, C.; ALFONSO MOLA, M. (2007): La Ruta Española a China. Madrid, Ediciones El Viso.

MCMAHON, K. (2002): The Fall of the God of Money. Opium Smoking in Nineteenth-Century China. Lanham (MD), Rowman \& Littlefield Publishers.

MCMASTER, J. (1959): "Aventuras asiáticas del peso mexicano". Historia Mexicana, 8/3, pp. 372-399.

MORSE, H. B. (1926): The Chronicles of the East India Company trading to China, 1635-1834. Oxford, Clarendon Press.

MUI, H.-ch.; MUI, L. H. (1984): The Management of Monopoly. A Study of the English East India Company's Conduct of Its Tea Trade, 1784-1833. Vancouver, University of British Columbia Press.

NEWMAN, R. K. (1995): "Opium smoking in Late Imperial China: A Reconsideration". Modern Asian Studies, 29/4, pp. 765-794.

NG C.-k. (1983): Trade and Society. The Amoy Network on the China Coast, 1683-1735. Singapur, Singapore University Press.

PARRÓN SALAS, C. (1995): De las Reformas borbónicas a la República: El Consulado y el comercio marítimo de Lima, 1778-1821. San Javier, Imprenta de la Academia General del Aire.

PAULÈS, X. (2011): L'opium. Une passion chinoise (1750-1950). París, Payot.

PÉREZ LECHA, M. (2014): "Los últimos años del Galeón de Manila. El ocaso de un modelo colonial hispano en el Pacífico, 1785-1821". Tesis doctoral, Castellón de la Plana, Universitat Jaume I.

PERMANYER UGARTEMENDIA, A. (2012): "Españoles en Cantón: los Diarios de Manuel de Agote, primer factor de la Real Compañía de Filipinas en China (1787-1796)". Itsas Memoria. Revista de Estudios Marítimos del País Vasco, 7, pp. 523-546.

- (2013): "La participación española en la economía del opio en Asia Oriental tras el fin del Galeón". Tesis doctoral, Barcelona, Universitat Pompeu Fabra. 
- (2014): "Opium after the Manila Galleon: The Spanish involvement in the opium economy in East Asia (1815-1830)". Investigaciones de Historia Económica. Economic History Research, 10, pp. 155-164.

- (en prensa): "Los Diarios de Manuel de Agote, factor de la Real Companía de Filipinas en China", en BERNABEU ALBERT, S.; MENA GARCÍA, C.; LUQUE AZCONA, E. J. (Eds.), Filipinas y el Pacífico: Nuevas miradas, nuevas reflexiones. Sevilla, Secretariado de Publicaciones de la Universidad de Sevilla.

PRAKASH, O. (1987): "Opium monopoly in India and Indonesia in the eighteenth century". The Indian Economic and Social History Review, 24, pp. 63-80.

QUIASON, S. D. (1966a): "The Sampan Trade, 1570-1770", en FELIX, A., Jr., (Ed.), The Chinese in the Philippines. Manila y Nueva York, Solidaridad Publishing House.

- (1966b): English "country trade" with the Philippines. Manila y Nueva York, Solidaridad Publishing House.

RUIZ DE GORDEJUELA, J. (2006): La expulsión de los españoles de México y su destino incierto, 1821-1836. Sevilla, Consejo Superior de Investigaciones Científicas-Secretariado de Publicaciones de la Universidad de Sevilla. TRACY, N. (1995): Manila Ransomed. The British Assault on Manila in the Seven Years War. Exeter (Reino Unido), University of Exeter Press.

TROCKI, C. A. (1999): Opium, Empire and the Global Political Economy. A study of the Asian opium trade, 1750-1950. Londres y Nueva York, Routledge.

VALDÉS LAKOSWKY, V. (1987): De las minas al mar. Historia de la plata mexicana en Asia, 1565-1834: México, D. F., Fondo de Cultura Económica. VAN DYKE, P. A. (2005): The Canton Trade. Life and Enterprise on the China Coast, 1700-1845. Hong Kong, Hong Kong University Press.

- (2011): Merchants of Canton and Macao. Politics and Strategies in Eighteenth-Century Chinese Trade. Hong Kong, Hong Kong University Press.

ZHAO, G. (2013): The Qing Opening to the Ocean. Chinese Maritime Policies, 1684-1757. Honolulu (HI), University of Hawai'i Press.

ZHENG Y. (2005): The Social Life of Opium in China. Cambridge, Cambridge University Press.

- (2011): China on the Sea. How the Maritime World Shaped Modern China. Leiden, Brill. 